\title{
Aproximación a la representación de personajes de grupos de la diversidad sexual
}

\section{Approach to the representation of characters of sexual diversity groups}

\author{
Sergio Palomino Gámez ${ }^{1}$ \\ José Carlos Vázquez Parra ${ }^{2}$
}

\section{Resumen}

El objetivo de este artículo ha constituido una aproximación a la manera en que la representación de personajes del colectivo LGBTI+ se ha dado en las últimas décadas en los medios audiovisuales. La intención de este análisis es señalar el impacto que, una representación sesgada o estereotipada puede tener en la constitución de imaginarios incorrectos o limitados que detonan situaciones de discriminación hacia este grupo poblacional. La reflexión se centra en la necesidad de poner atención en la responsabilidad que significa para los generadores de contenidos, el construir y presentar personajes lo más auténticos y apegados a la realidad posible, considerando que esto es parte de la responsabilidad social que tienen hacia sus consumidores.

Palabras clave: Estereotipos; Estudios de Género; Discriminación; Imaginarios Culturales; LGBTI+.

\section{Abstract}

The objective of this article has been an approximation to the way in which the representation of characters from the LGBTI + collective has occurred in the last decades in the audiovisual media. The intention of this analysis is to point out the impact that a biased or stereotyped representation can have on the constitution of incorrect or limited imaginaries that trigger situations of discrimination towards this population group. The reflection focuses on the need to pay attention to the responsibility that means for content generators, to build and present characters as authentic and attached to reality as possible, considering that this is part of the social responsibility they have towards their consumers

Keywords: Stereotypes; Gender Studies; Discrimination; Cultural Imaginaries; LGBTI+.

\footnotetext{
1 Maestro en Comunicación con especialidad en Producción Audiovisual. Profesor de Cátedra del Departamento de Español, Literatura, Ciencias Sociales y Humanidades de la Prepa Tec Guadalajara. Correo: spalomino@tec.mx Orcid: https://orcid. org/oooo-0oo1-7144-3173

2 Doctor en Estudios Humanísticos con especialidad en Ética. Profesor investigador de la Escuela de Humanidades y Educación del Tecnológico de Monterrey, Región Occidente. Correo: jcvazquezp@tec.mx Orcid: https://orcid.org/oooo-0o01-9197-7826
}

Recibido: 08/01/2021 - Aprobado: 05/02/2021 


\section{Introducción}

Cuando se habla del impacto de los medios de comunicación en la vida de las personas, el tema no sólo se relaciona con la calidad o con la medición de preferencias del público, sino también, y de forma contundente, en la manera en que los contenidos pueden influir en lo que las personas creen, deciden o hacen. Esto, ha llevado a que durante años se estructuren sistemas que clasifiquen la pertinencia de ciertos públicos para determinados contenidos, lo cual refleja el interés que socialmente se tiene sobre el contenido que consume la audiencia.

Desde un enfoque asertivo, también se ha reflexionado sobre cómo los medios de comunicación, pueden influir considerablemente en la percepción que tienen las personas acerca de un lugar o de un individuo, como se hace en las campañas políticas, entretejiéndose una relación entre los sistemas de creencias y el imaginario cultural de una sociedad en concreto (Le Bon, 2018).

Objetivo de este artículo fue analizar lo relacionado a la representación de personajes en medios audiovisuales, desde la premisa de que la buena o mala representación de ciertos grupos minoritarios, como es el colectivo LGBTI+ (siglas de lesbiana, gay, bisexual, transgénero, transexual, travesti, intersexual y queer), puede influir en la percepción de la población en general, tanto positiva como negativamente. Como conclusión, se reflexiona sobre la responsabilidad de los generadores de contenidos, planteando que apegarse a las representaciones auténticas es un principio de responsabilidad social

\section{Desarrollo}

\section{Representación y estereotipos}

Ferrés (1996), consideraba que la televisión permitía legitimar modelos del mundo, construyendo en la audiencia una significación de la realidad, atribuyéndole nuevos significados desde comunicaciones pre-lingüísticas inadvertidas, ya que, en estos contenidos, no es necesario decir muchas cosas para explicar lo que se quiere. Así, la televisión y en su momento las plataformas de contenido audiovisual son considerados como un pilar importante en el desarrollo intelectual, conductual y emocional, ya que son medios de exposición de realidades, que, con una exposición alargada, pueden dotar de argumentos, correctos o inadecuados, para la constitución de creencias en el auditorio.

Se entiende por un estereotipo a aquella percepción exagerada y poco detallada que se tiene de una cosa, persona o grupo de personas que comparten ciertas características, cualidades y habilidades, a partir, usualmente, de juicios de valor previamente 
atribuidos y construidos a partir de ideologías y creencias, que no siempre son correctas (Lamorena, Alcántara y López, 2019).

De esta forma, los estereotipos pueden dar lugar a visiones simplistas o poco realistas de las cosas o las personas, distorsionando la realidad y lo que llega a percibirse de la misma, lo cual, por tener esta argumentación cognitiva, afectiva y comportamental, suele verse con naturalidad, lo que lleva a que sea compleja su identificación. Usualmente, los estereotipos se generan del desconocimiento que se tiene acerca de algo que se considera extraño, mismo que resulta amenazante y, por ende, justifica el desarrollo de reacciones y respuestas de rechazo (Francisco y Moliner, 2011).

En cuanto a los medios de comunicación, el estudio de estereotipos es un modo útil de estudiar el nexo entre imagen e ideología. Los estereotipos indican que consideramos típico o característico de un grupo y nos ofrecen ejemplos concretos y accesibles de la ideología en la práctica. Cuando examinamos estereotipos de categorías sociales como las mujeres, los jóvenes, los ancianos y grupos étnicos en los medios, analizamos costumbres culturales que tienen ramificaciones políticas y sociales significantes (Massanet, 2016).

Masterman (1993) apunta que, los medios son importantes moldeadores de las percepciones e ideas, siendo elementos de concienciación que no sólo proporcionan información acerca del mundo sino maneras de verlo y entenderlo. Morduchowicz y Munzi (2003) señalan, precisamente, cómo las representaciones son herramientas del discurso en tanto seleccionan, jerarquizan, reorganizan, matizan, destacan, subrayan y fragmenta la realidad.

Los estereotipos de grupo son una de las formas más frecuentes de representación en los medios de comunicación, lo cual implica una imagen mental simplificada y que no necesariamente se encuentra apegada a la realidad. Lamentablemente, estas imágenes suelen darse de forma tan reiterada que llegan a sumarse al imaginario cultural, por lo que cuando un sujeto pertenece a este grupo suele difuminarse su individualidad predominando el estereotipo del grupo, lo cual es una forma clara de discriminación y de normalización de prejuicios. Al fin de cuentas, los estereotipos presentan una percepción de las cosas o los grupos que poco o nada tiene que ver con la realidad y el respeto a las diferencias, siendo los medios de comunicación, un espacio donde usualmente se proyectan estas construcciones sesgadas (Francisco y Moliner, 2011).

\section{Representación de los grupos de la diversidad sexual en los medios}

Los discursos mediáticos del siglo pasado solían estar caracterizados por lo que se denominaba la triada de la comunicación, la cual, implicaba la necesidad de que todo contenido producido fuera revisado tanto por el Estado, la ciencia y la religión (Bazán, 2010). Esto, llevó que gran parte del siglo XX y hasta 1983, la orientación sexual 
no fuera vista como parte de una diversidad o tuviera espacio para las diferencias, pues todo aquello que no se apegaba al parámetro heteronormativo y reproductivo, era considerado una desviación, por lo cual, su visibilización estaba prohibida. Tradicionalmente, la comunidad LGBTI+ es uno de los grupos minoritarios usualmente menos considerado por la industria mediática, y cuando se les incluye, suele ser desde una visión sesgada y altamente estereotipada (Francisco y Moliner, 2011).

El único espacio en el que el homosexualismo tuvo cabida fue en lo relacionado con la llamada nota roja, la cual se caracterizaba por los constantes asesinatos de miembros de esta comunidad (Butler, 2002). Sin embargo, la visión con la que se cubrían estas noticias era claramente acusatoria de las víctimas, acompañando las narrativas con calificativos denigrantes y que señalaban su promiscuidad o la amoralidad de su comportamiento (Sabsay,2011).

Sin embargo, y con el detonar del activismo LGBTI+ a inicios de la década de los 70 los medios de comunicación no pudieron mantenerse ajenos a la presión y cuestionamiento, por parte del colectivo, de esta visión heteronormada de la información. Así, aunque la sanción por parte de las instituciones continuaba estando presente, algunos medios alternativos se arriesgaban a abrir espacios para el dialogo y reflexión acerca de la lucha por la igualdad de este grupo, aunque usualmente eran discusiones que iniciaban y terminaban al momento mismo de la publicación (Butler, 2002).

De manera concreta, en la región latinoamericana, la sombra ejercida por las dictaduras militares fue algo ante lo cual los medios de comunicación no pudieron sobreponerse con facilidad, teniendo restricciones institucionales fuertemente marcadas hacia lo que se podía o no publicar, siendo todo lo relacionado con la diversidad sexual, algo prohibido por ser considerado inmoral y escandaloso para las masas (Bazán, 2010).

Conforme avanzaron los años, la presión social también fue mayor, detonando diferentes oportunidades de visibilización en los medios audiovisuales. En 1972, la serie norteamericana That Certain Summer presentaba la historia de un padre divorciado que ocultaba el hecho de que era gay a su hijo, lo que vino seguido por la adaptación del libro Quentin Crisp en la sería de 1976 The Naked Civil Servant, que retrataba la vida en San Francisco. La década de los 80 y la crisis del VIH influyo a incluir este tipo de conversaciones en sus temáticas, como fue el filme de la NBC An Early Frost (Fellner, 2017).

En Latinoamérica, los primeros intentos de visibilización a finales de la década de los 80, con dramas televisivos como la novela Rossé de 1986 en donde se incluía un personaje homosexual, o bien, en la telenovela Sin Marido de 1988, en donde se planteaba la posibilidad del amor entre dos mujeres. Claro está que el abordaje que se hacía era sumamente sutil e indirecto, por lo que gran parte de lo que se sabía o 
entendía de los personajes, era correspondiente más a la representación que a una trama que directamente mencionase estos temas (Rubio, R., 2017).

Lamentablemente, estas primeras manifestaciones en los medios tuvieron que venir aparejadas a una situación sanitaria desastrosa para la comunidad en el ámbito internacional, puesto que a la par de esta visibilización se desarrollaron fuertes señalamientos y estereotipos acerca del estilo de vida homosexual y su relación con el VIH-Sida y otras enfermedades (Reguillo, R., 2008). En las narrativas mediáticas de la época, el carácter invisible comenzó a ser reemplazado por modelos de segregación y discriminación aunados a una visibilización máxima.

Durante los 9o, fue aumentando considerablemente la exposición y presencia mediática de la comunidad LGBTI+; sin embargo, en la mayoría de las ocasiones, esto venía apegado a una visión negativa del grupo, las personas y su estilo de vida. En consecuencia, la visibilidad operaba como liberadora, pero a su vez como operadora de estigmatización, reproduciendo patrones de desigualdad existentes, mezclando discursos que promovían discursos de carácter antidiscriminatorio con otros de tono segregante, excluyente y de juicio moral (Reguillo, R., 2008). Aun así, a mediados de los 9o, la presencia de personajes de hombres gays en la televisión fue cada vez más usual. Sin embargo, se hace evidente que estos personajes, en su totalidad en caracteres secundarios, perpetuaban los estereotipos negativos, siendo representaciones construidas bajo la visión afeminada, delicada, picara y con una actividad asociada a las mujeres (Rubio, 2017). Algo cotidiano, es que estos personajes, representados por hombres heterosexuales, se manejaban bajo un velo de comedia y burla, alejándolos de la realidad y teniendo una visión heteronormativa de la trama de la historia. Esta situación, aunque visibilizaba a la comunidad, no mostraba su realidad, siendo incluso distractores de lo que verdaderamente implica la vida de una persona homosexual.

Esto se presentaba de igual manera en el medio cinematográfico, en el que estudios como el de Shirley Steinberg sobre la película In\&Out critican el frecuente uso de estereotipos homosexuales en el cine de Hollywood, el cual recurre a una visión sesgada y asexuada de los personajes (Steinberg, 2005). Lo interesante de esto último, es que esta visión de invisibilización de la sexualidad era un medio de difuminar lo relacionado a la vida sexual de los personajes, tratando de hacer que la representación de homosexuales en la televisión cumpliera, por un lado, con el reclamo social de visibilización, pero por el otro, no fuera una imagen desafiante para el público heterosexual (Matí, 2011).

Sin lugar a duda, la segunda mitad de la década de los 90 marcó un parte aguas sobre lo que se venía ofreciendo en cuanto a productos audiovisuales con temática LGBTI+, ya que series como Ellen, quien es la primera protagonista dentro de una trama que reconoce su homosexualidad y sale del closet en 1997, y el estreno al año siguiente de la comedia Will \& Grace que presenta una historia completa en torno a 
la vivencia de un hombre homosexual, serían los avances más significativos de esta comunidad en los medios de comunicación (Matí, 2011). Sin embargo, amplias criticas seguían dándose acerca de la representación de los personajes en estas producciones, ya que, aunque las historias versaban primordialmente en personajes de la comunidad LGBTI+, sus historias seguían siendo sumamente cautas y sutiles en cuanto a su estilo de vida (Fujita, 2018).

En 1999 Channel 4 lanza Queer as Folk, serie británica que vendría a reconfigurar lo que el imaginario televisivo venía manejando hasta ese momento sobre el estilo de vida homosexual. A diferencia de los personajes homosexuales asexuados que proponía la televisión norteamericana, Queer as Folk mostraba con crudeza y realidad, la vida de un grupo de homosexuales en una zona gay de Manchester, ampliando la visión no solo a las cuestiones sexuales de sus personajes, sino también, a la posibilidad de hablar sobre relaciones de pareja, sus familias y todo lo relacionado con momentos de vida como los procesos de salir del closet o de estar enfermo de VIH (Billingham, 2017). Fue tal el impacto que llegó a tener esta producción, que un año después, Showtime estaría haciendo una adaptación para los Estados Unidos (Hart, 2016).

Así, el inicio del siglo pareció abrir pauta a una exigencia social por no solo visibilizar a los miembros de la comunidad LGBTI+, sino también el hacerlo de una manera autentica y realista. Lamentablemente, no todos los miembros de este colectivo gozaron del mismo impulso y presencia en estos esfuerzos de conquista de espacios mediáticos, pues, aunque los hombres homosexuales estaban cada vez más presentes en los medios, esto no se dio de igual manera con las mujeres lesbianas, y mucho menos, con la comunidad trans, la cual ha tenido una presencia casi nula hasta hace algunos pocos años (McLaughlin y Rodríguez, 2017).

\section{La representación lésbica en los medios. Un tema paralelo}

A diferencia del proceso de visibilización de los personajes masculinos homosexuales en los medios de comunicación, la invisibilización lésbica parece surgir incluso desde la falta de una percepción de la realidad de este grupo. Los estereotipos existentes, no han sido ni siquiera generados como una exageración o distorsión de la realidad, sino más bien, como parte del imaginario heterosexual, que inserta a las lesbianas en la industria de la pornografía, la explotación sexual y las fantasías (Esteban, 2004).

Lo anterior, no solo perjudica al desarrollo de una imagen de grupo y la visibilización de éste, sino que incluso impacta en la sociedad en general al ignorar parte de la diversidad del colectivo LGBTI+. De manera concreta para las mujeres lesbianas, estos estereotipos pueden llegar a afectar la identidad que se tiene de ellas mismas, con creencias que poco abonan a una idea realista de lo que es la vida de una mujer homosexual (Turkle, 1997). 
Así, en la construcción mediática, las mujeres lesbianas son doblemente discriminadas por pertenecer a dos minorías, sufriendo una invisibilización tal que se perpetuaba la discriminación (Platero, 2008). Mientras en la década de los 70 ya comenzaba a haber representación de hombres homosexuales en la televisión o el cine, los personajes lésbicos eran casi inexistentes, y cuando se mostraba una relación entre dos mujeres, usualmente tenía un enfoque relacionado con la locura o la perversión (Gauntlett, 2002).

En una de las pocas películas que abordaba esta cuestión, "The hour of the children" (1961), el personaje interpretado por Audrey Hepburn dice "there must be some place where they could go" (debe existir un lugar donde podamos ir); pero, parecía que realmente las lesbianas no tenían un espacio para vivir. Hay que esperar hasta el estreno de "Desert Hearts" en 1985 para encontrar la primera película con un final feliz entre una pareja de mujeres enamoradas (González, 2011).

En televisión, los personajes de lesbianas y bisexuales tampoco han tenido suerte. El primer personaje que aparecía de manera continuada de una lesbiana aparecería en All My Children en 1970, mismo que estaba fuertemente estereotipado. El primer beso entre dos mujeres se dio en 1986 en la serie L.A. Law, aunque éste tenía mayor relación con una fantasía heterosexual, que verdaderamente una cuestión lésbica. Hubo que esperar hasta 1996, en la serie Relativity para poder ver el primer beso entre dos personajes femeninos bisexuales. Y hasta el 2003, es decir, 34 años después del inicio del activismo LGBTI+, para poder ver la primera escena de sexo entre dos mujeres en una televisión abierta, en la serie Buffy. Todo esto demuestra que esta comunidad ha estado excluida de las producciones mediáticas hasta hace muy poco (Francisco y Moliner, 2011).

En cuanto a series que reflejaban la vida de mujeres lesbianas, fue Ellen la que comenzó este proceso de visibilización en 1994, sin embargo, fue la serie Queer as Folk, la que presentaba una representación considerada muy autentica de la vida de una pareja de lesbianas entre su reparto principal. La aceptación fue tan considerable, que en 2004 se produce The $L$ Word, primera serie en el que todos sus personajes principales eran lesbianas y bisexuales, buscando no solo la visibilidad del estilo de vida lésbico y de la propia diversidad existente en el grupo, sino también, el empoderamiento de la mujer homosexual en la lucha por los derechos del colectivo LGBTI+ (Francisco y Moliner, 2011). Lamentablemente, la serie recibió críticas por consolidar otro tipo de estereotipos, ya que la mayoría de sus protagonistas eran mujeres blancas, exitosas, delgadas y de clase alta (Bartley, 2016). Según GLAAD, aunque hay una mayor visibilidad LGBTI+ en las ficciones seriadas, el número de personajes femeninos sigue siendo considerablemente inferior que el de varones (Padilla, 2018). 


\section{La invisibilización trans}

Respecto a esta parte del colectivo LGBTI+, la presencia de personas trans en la televisión inició primordialmente a partir de la representación de personajes masculinos travestidos, los cuales, eran usualmente ligados al mundo del espectáculo o el comercio sexual. Lo anterior, desarrolló fuertes estereotipos que ridiculizaban o estigmatizaban a esta población, reforzando prejuicios existentes (Grimson, 2011).

La primera vez que la televisión americana "recreo" a un personaje transgénero, lo hizo en 1965 en el seriado dirigido por Alfred Hitchcock que llevaba el nombre de The Alfred Hitchcock Hour, el cual era un personaje sumamente estereotipado y básico (Bermúdez, 2017). Estos inicios, muestran la configuración que la transexualidad y el trasngeneralismo tenía en el imaginario social de la época, lo cual estaba lleno de confusiones sobre la diferente terminología (Padilla, 2018).

En los 9o, aunque la representación de personas transexuales fue en aumento no hubo cambios significativos en cuanto la manera en que se mostraban los personajes, siguiendo esta visión transfóobica y limitante. Los personajes eran primordialmente incluidos en series de comedia, relacionándolos nuevamente con el travestismo, la prostitución y fomentando una visión sesgada de este colectivo.

Tuvieron que pasar décadas, para la presencia de la comunidad trans fuera reconocida dentro de la televisión con roles verdaderamente profundos. Ejemplos como Laverne Cox en la serie Orange is the New Black, fue tan representativa que la hizo ganadora de un Emmy, detonando la presencia del colectivo en la televisión norteamericana. En 2008, la serie de realidad America's Next Top Model incluyó a la primera aspirante transexual. De manera más profunda, programas como I am Jazz y I am Cait, mostraban la realidad que viven las personas trans durante todo su proceso de aceptación, lo cual, consideraba temáticas relacionadas con los procesos de cambio de sexo genital (Carpenter, 2017).

\section{La Responsabilidad de los Generadores de Contenidos}

Un punto fundamental y de suma importancia para esta reflexión, es considerar que la invisibilización del colectivo LGBTI+, su sesgada representación y los estereotipos que se han perpetuado por ello, incumben en gran medida a los generadores de contenido, quienes hace apenas pocos años han visto la importancia de hacer representaciones auténticas, lo cual, es una responsabilidad social que tienen hacia sus consumidores.

Para Acción Empresarial (2003), toda organización que ofrece productos a las nuevas generaciones debe comprender que sus acciones tienen un claro impacto social, valorando lo que sucede más allá de sus límites físicos, previendo las necesidades y 
legítimas demandas de sus grupos de interés, entre los cuales están sus consumidores/usuarios, así como la agenda social internacional que promueve el bienestar y el desarrollo humano.

Según Kotler y Zaltman (1971), los medios de comunicación tienen una marcada obligación informativa, llegando a influir en la aceptación de ideas sociales a partir de sus productos. Por ende, la legitimidad y objetividad de la información que se brinda es una obligación que tiene toda empresa de comunicación, incluidas las relacionadas a la generación de contenidos audiovisuales, procurando respetar una visión autentica de la realidad, así como evitar tendencias apegadas a ideologías, creencias o intereses particulares (Capriotti, 2007). El derecho que tiene la sociedad en general de acceder a información confiable no es una opción ni para el comunicador, ni para el productor de contenidos, o la empresa de medios, por ser una responsabilidad que viene apegada a la razón de ser de los medios de comunicación (Cortina, Martínez, y Siurana, 2005).

Desde la perspectiva de Lotero, Romero y Pérez (2018), es reprochable que las empresas de comunicación transmitan información que exagera o tiende a ser tendenciosa, ya que esto, llega a confundir a la población, alejándola de una visión objetiva de la realidad. Para Arredondo, Rosas y Villa (2011), la responsabilidad social de la industria de las comunicaciones también debe incluir el velar por el desarrollo de una conciencia colectiva, ya que es inevitable el impacto que estas llegan a tener en el imaginario social.

En cuanto a lo relacionado con la representación de personajes, y considerando a estos como parte del contenido que se produce y oferta, es necesario que los productores y generadores de ideas, prevean la necesidad de pasar por el mismo filtro de objetividad y autenticidad, ya que la presencia de estereotipos o visiones sesgadas sobre un grupo representado, es tan grave como lo sería presentar información falsa o alterada sobre una realidad o cuestión histórica (Romero, Moreno y Toukoumidis, 2015).

Así, la responsabilidad social de la industria de generación de contenidos debe enfocarse en la necesidad de responder por lo que produce, al igual que lo haría cualquier medio de comunicación de corte informativo, al considerar el impacto que puede llegar a tener el producto que ofrece para su consumo social. Hablar de contenido en una obra audiovisual, incluye no únicamente la trama o el contexto en el que la historia se desenvuelve, sino también, la relación que la misma tiene con los personajes que se representan y sus propias historias de vida. El personaje, bajo una visión psicológica, es un ente complejo que, aunque ficticio, responde a su propia realidad, debiendo esta ser considerada al momento de la generación de todo el producto audiovisual. 


\section{Conclusión}

Los procesos de identificación de los consumidores de productos audiovisuales implican algo más allá que la trama o la historia, sino también, los personajes y la representación que se pretende hacer con ellos. Por ende, es responsabilidad de los generadores de contenido el poder reconocer el impacto que sus productos pueden tener, al reafirmar o perpetuar modelos sesgados o estereotipados de información. Lógicamente, esto conlleva el que la industria audiovisual, deba velar por la oferta de contenidos que eviten esta visión alterada de las cosas, los entornos y las personas, ya que, aunque sea de manera indirecta, la generación de contenidos tiene una función informativa.

Hablar de una verdadera responsabilidad social hacia la diversidad, debe comenzar con el respeto de la comunidad a partir de la razón de ser de la organización, por lo que para nada sirve una responsabilidad en proyectos externos que no incluyan la generación de productos y servicios responsables. Es menester reconocer que el desarrollo de una cultura de inclusión y diversidad comienza con la modificación de las creencias tradicionales y de corte heteronormativo que socialmente se vienen arrastrando, siendo los medios de comunicación, a través de la generación de contenidos auténticos, una excelente forma de romper estereotipos. Así, la representación autentica y realista de personajes, sobre todo aquellos que forman parte de cierto colectivo, es un tema de suma relevancia al momento de la valoración de la calidad de un producto audiovisual y su posible oferta, lo que incluye un claro compromiso y responsabilidad por parte de los generadores de contenidos.

\section{Lista de referencia}

Acción Empresarial (2003). El ABC de la responsabilidad social empresarial en Chile y el Mundo. Recuperado el 02 de 05 de 2016, de http://www.javeriana.edu.co/documents/245769/3057616/El_ABC_de_la_RSE_en_Chile_y_el+mundo_2003. pdf/823c876b-779d-4a7b-b78a-6d6043815c63

Arredondo Trapero, F., Rosas Ferrer, J., y Villa Castaño, L. (2011). Comportamiento ciudadano organizacional y RSE. Cuadernos de Administración, 24(43), 221239.

Bartley, M. (2016). Victory stands on the back of sacrifice: The odies and body politics of lesbian and bisexual women on TV. Atrium. Writing Studies Program, 236262.

Bazán, O. (2010). Historia de la homosexualidad en la Argentina. De la conquista de América al siglo XXI. Buenos Aires: Marea.

Bermúdez, J. (2017). Psycho Killers, Circus Freaks, Ordinary People: a brief history of the representation. Boston: Océanide. 
Billingham, P. (2017). Can kinky sex be politically correct? Quees as Folk ang the geoideological inscription of gay sexuality. Manchester: Manchester University Press.

Butler, J. (2002). Cuerpos que importan. Sobre los límites materiales y discurivos del sexo. Buenos Aires: Paidós.

Capriotti, P. (2007). La responsabilidad social corporativa de las empresas españolas en los medios de comunicación. ZER: Revista de estudios de comunicación, 12(23), 61-74.

Carpenter, C. (2017). The T in LGBT: Orange is the New Black and trasgender issues in corrections. Corrections Today, 79, 52-56.

Cortina, A., Martínez, E., y Siurana, J. (2005). Ética de las profesiones. Monterrey: ITESM.

Esteban, M. (2004). Antropología del cuerpo. Género, itinerarios corporales, identidad y cambio. Barcelona: Bellaterra.

Fellner, A. (2017). Trans television culture. Queer politics, gender fluidity and quality TV. Boston: Oceánide.

Ferrés, J. (1996). Televisión subliminal. Socialización mediante comunicaciones inadvertidas. Barcelona: Paidós.

Francisco, A., y Moliner, L. (2011). Porque la visibilidad importa. Una propuesta para trabajar la diversidad sexual en la escuela a través de la educación en medios. Revista Educación Inclusiva. Revista Educación Inclusiva, 12(4), 1-12.

Fujita, A. (2018). Changing Perception of LGBT people through performances. Journal of Urban Culture Research (17), 54-71.

Gauntlett, D. (2002). Media, Gender and Identity: An introduction. New York: Routledge.

González, C. (2011). Visibilidad y diversidad lésbica en el cine español. Icono 14. Revista de Comunicación y Nuevas Tecnologías, 9, 221-255.

Grimson, A. (2011). Los límites de la cultura. Críticas de las teorías de la identidad. Buenos Aires: Siglo XXI.

Hart, K. (2016). Queer TV in the 21st Century. Essays on Broadcasting from Taboo to Acceptance. Chicago: McFarland. 
Kotler, P., y Zaltman, G. (1971). Social Marketing: An approach to planned social change. Journal of marketing, 3-12.

Lamorena, Á., Alcántara, J., y López, M. (2019). Sexo, violencia y estereotipos en el band equity de una serie. El caso de juego de tronos. Cuadernos de gestión, 19(1), 15-40.

Le Bon, G. (2018). Psicología de las masas. Madrid: Verbum.

Lotero, G., Romero, L., y Pérez, M. (2018). Fact-checking vs. Fake news: Periodismo de confirmación como componente de la competencia mediática contra la desinformación. Index Comunicación, 8(2), 295-316.

Massanet, M. (2016). Pervivencia de los estereotipos de género en los hábitos de consumo mediático de los adolescentes: drama para las chicas y humor para los chicos. Cuadernos, 39, 39-53.

Masterman, L. (1993). La enseñanza de los medios de comunicación. Madrid: Ediciones la Torre.

Matí, E. (2011). Homosexualidad, infancia y animación: Del nacimiento de Pebbles Picapiedra a la adopción de Ling Bouvier. Con A de animación, 22(1), 2-15.

McLaughlin, B., y Rodríguez, N. (2017). Identifying with a stereotype: The divergent effects of exposure to homosexual television characters. Journal of homosexuality, 64(9), 1196-1213.

Morduchowicz, R., \& Minzi, V. (2003). Claves para pensar el trabajo con la prensa gráfica en la escuela. Madrid: Octaedro.

Padilla, O. (2018). Estudio de los personajes LGBTI en la ficción seriada norteamericana. Barcelona: Universitat Autónoma de Barcelona.

Platero, R. (2008). Las lesbianas en los medios de comunicación: madres, folclóricas y masculinas. En R. Platero, Lesbianas. Discursos y representaciones (págs.307-338). Madrid: Melusina.

Reguillo, R. (2008). Políticas de la (in) visibilidad. La construcción social de la diferencia. Buenos Aíres: FLACSO.

Romero, L., Moreno, P., y Toukoumidis, A. (2015). Estereotipos, tópicos y lenguaje de la programación sensacionalista en la televisión: programa «Corazón» de TVE. Alteridad, 10(1), 31-43. 
Rubio, R. (2017). La telenovela como tecnología de género y la codificación de la disidencia sexual masculina en Chile. Santiago: Universidad de Chile.

Sabsay, L. (2011). Fronteras sexuales. Espacio urbano, cuerpos y ciudadanía. Buenos Aires: Paidós.

Steinberg, S. (2005). Del armario al corral: nuevos estereotipos en In \& Out. En S. Talburt, \& S. Steinberg, Pensando Queer. Sexualidad, cultura y educación (págs. 163-170). Barcelona: Graó.

Turkle, S. (1997). La vida en la pantalla: la construcción de la identidad en la era de internet. Barcelona: Paidós 